\title{
A Proof of Concept for 3D Printing of Solid Lipid-based Formulations of Poorly Water-Soluble Drugs to Control Formulation Dispersion Kinetics
}

Kapilkumar Vithani ${ }^{1}$, Alvaro Goyanes ${ }^{2}$, Vincent Jannin ${ }^{3,4}$, Abdul W. Basit $^{2}$, Simon Gaisford ${ }^{2}$, Ben J. Boyd ${ }^{1,5^{*}}$

${ }^{1}$ Drug Delivery, Disposition and Dynamics, Monash Institute of Pharmaceutical Sciences, Monash University (Parkville Campus), Parkville, Victoria 3052, Australia

${ }^{2}$ UCL School of Pharmacy, University College London, 29-39 Brunswick Square, London, WC1N 1AX, UK

${ }^{3}$ Gattefossé SAS, 36 Chemin de Genas, 69804 Saint-Priest, France

${ }^{4}$ Lonza Pharma \& Biotech, Parc d'Innovation, Rue Tobias Stimmer - BP 30442, 67412 Illkirch Graffenstaden, France

${ }^{5}$ ARC Centre of Excellence in Convergent Bio-Nano Science and Technology, Monash University (Parkville Campus), Parkville, Victoria 3052, Australia

*Corresponding author: Ben J. Boyd Email: ben.boyd@monash.edu; Phone: +61399039912 


\section{Abstract}

Purpose: The use of three-dimensional printing (3DP) in the development of pharmaceutical dosage forms is growing rapidly. However, the research is almost exclusively focussed on polymer-based systems with very little reported on 3D printing of lipid-based formulations. Thus, the aim of the work was to explore the feasibility of 3DP technology to prepare solid lipid-based formulations. Here, 3DP was applied for the preparation of solid selfmicroemulsifying drug delivery systems (S-SMEDDS) with defined surface area to volume $(\mathrm{SA} / \mathrm{V})$ ratios.

Methods: The S-SMEDDS formulations, comprised of Gelucire ${ }^{\circledR} 44 / 14$, Gelucire $^{\circledR} 48 / 16$ and Kolliphor ${ }^{\circledR} \mathrm{P} 188$ were loaded with fenofibrate or cinnarizine as model drugs. The formulations were printed into four geometrical shapes - cylindrical, prism, cube and torus, and compared to a control cube manually prepared from bulk formulation.

Results: The printing process was not significantly affected by the presence of the model drugs. The as-printed S-SMEDDS formulations were characterised using differential scanning calorimetry and wide-angle X-ray scattering. The kinetics of dispersion depended on the SA/V ratio values. The digestion process was affected by the initial geometry of the dosage form by virtue of the kinetics of dispersion of the dosage forms into the digestion medium.

Conclusions: This proof of concept study has demonstrated the potential of 3DP for the development of customised S-SMEDDS formulations without the need for an additional carrier or additive and with optimisation could elaborate a new class of dosage forms based on 3D printed lipids.

Keywords: 3D printing, solid self-microemulsifying drug delivery system (S-SMEDDS), geometrical shapes, surface area to volume ratio value, kinetics of dispersion and digestion 


\section{Introduction}

The co-administration of poorly water-soluble drugs with lipid-based formulations generally enhances the oral bioavailability of these compounds through a number of mechanisms including maintaining the drugs in the solubilised state, modifying gastric emptying and by changing the drug distribution post absorption (1-6). The enzymatic digestion of lipids leads to the formation of digestion products such as monoglycerides and free fatty acids. These digestion products interact with endogenous components (i.e. bile salt, phospholipid and cholesterol) and form a variety of colloidal structures that can act as a transport medium for the absorption of lipophilic drugs.

In recent years, self-microemulsifying drug delivery systems (SMEDDS), comprising of oil, surfactant, co-surfactant, drug and sometimes some quantity of co-solvent, received great interest from industry and academia $(2,3,7,8)$. Upon dispersion, these SMEDDS formulations form a kinetically stable oil-in-water $(\mathrm{O} / \mathrm{W})$ emulsions with small particle size under mild agitation in the gastrointestinal (GI) tract where the lipophilic drugs are solubilised into the very finely dispersed lipid droplets $(2,9-12)$. Generally, these liquid formulations are filled into soft or hard gelatin capsules for an ease of oral administration. Despite the benefits, a number of drawbacks associated with the liquid state of the formulations may lead to problems including dose uniformity, interaction of formulation components with the capsule shell (that can potentially change the taste perception of the filling and/or can lead to the softening of the capsule shell or leakage of the filling), portability and chemical stability of drug in the liquid dose form. These issues have limited the translation of SMEDDS formulations $(2,4,13,14)$. Therefore, transforming these liquid formulations into solid-based lipid formulations (SSMEDDS) has received an increasing amount of interest as a means of circumventing some of those issues.

Traditionally, S-SMEDDS formulations are prepared by using several solidification techniques such as adsorption to solid carriers (4), spray-drying (15), spray-cooling (16), melt granulation (17), supercritical fluid-based methods (18) and melt extrusion/extrusion spheronization (1922). However, most of these techniques require a large amount of solid-phase carrier such as silica, dextran, magnesium aluminometasilicate, lactose, cellulose etc., to adsorb the liquid formulation and to generate a solid dosage form (i.e. powder sachets, tablet or pellets). These additional carriers can lead to the issues of dose dilution, tolerability and toxicity $(23,24)$. The dilution of liquid formulations into these solid-phase carriers and other additional excipients 
sometimes makes it difficult to achieve the right dose for high dose drugs, and affect the formulation compressibility (23). Additionally, the reproducibility is not always optimal and the production times are relatively long. S-SMEDDS formulations also require a large amount of surfactants to facilitate the high surface area be in contact with the enzymes in order to achieve digestion and thereby solubilisation of poorly water-soluble drugs into the digestion products. However, the higher quantity of surfactants may cause problems with tolerability of the dosage form, increase the cost and complexity of the preparation process $(25,26)$. Thus, a means of producing a high surface area S-SMEDDS formulations without the need of an additional solid-phase carrier/co-solvent and high concentration of surfactants would be highly desirable.

3D printing (3DP) has received great interest across numerous fields including material science and pharmaceutical discipline (27-32). The recent approval of the first 3D printed orodispersible tablet, Spritam ${ }^{\circledR}$ (levetiracetam), in 2015 by the FDA (Food and Drug Administration, the United States) and the advancement of 3DP in manufacturing medicines have already proven the feasibility of 3DP as a key technology in manufacturing pharmaceutical solid dosage forms (33-36). In 3DP, the printer follows the computer-aided design (CAD) instructions and produces a 3D object by depositing successive layers of materials on the build plate. In the pharmaceutical field, 3DP offers the advantages of accurate control of the spatial distribution of the drug particles inside the dosage form, manufacturing of complex geometries, deposition of a very small amount of substances, reduced waste, rapid fabrication of various compositions for convenient screening activities and the capacity to produce a dose form with personalised dose strengths (36-42). The 3DP technique comprises of different technologies such as stereolithography (SLA), selective laser sintering (SLS), inkjet, fused deposition modeling (FDM), binder-jetting and semi-solid extrusion printing (43, 44). The primary difference between the technologies is specifically based on the mechanism of the layer formation process to form a 3D object. The feasibility of 3DP technologies in the pharmaceutical arena is well-reported in the literature and the technology has been utilised to develop fast-disintegrating tablets (42), time-controlled release tablets (45-47), fabricated sustained-release dosage forms (48), multi-active ingredient tablets (49), nanocapsule-loaded tablets (50), microneedles (51) and other modes of topical drug delivery systems (52). The technology offers an alternative route of controlling geometry and hence surface area (SA) and volume (V) to control the dispersion of the formulation and thereby dissolution of the drugloaded solid matrix, and present a straightforward and cost-effective way to customise the drug 
release profiles $(29,53)$. For example, Goyanes et al. prepared polymer-based pharmaceutical tablets in a diverse range of geometrical shapes using FDM 3DP technology and studied the impact of geometry on the drug release process (29).

However, in pharmaceutics, the interest in 3DP has been highly focussed on polymer-based drug delivery systems. To date, the literature on using 3DP for the development of lipid-based drug delivery systems is very limited and, to the best of our knowledge, there is no report of utilising 3DP for the development of solid SMEDDS formulations.

Consequently, the current study aimed to provide proof of concept that 3DP technology can be useful in the preparation of drug-loaded S-SMEDDS formulations without the need of a solidphase carrier, and 3DP can potentially provide control of geometry and thereby provide control over dispersion and digestion. The drug-loaded S-SMEDDS formulations containing Gelucire ${ }^{\circledR}$ 44/14 (GEL 44 as a core digestible lipid), Gelucire ${ }^{\circledR}$ 48/16 (GEL 48 as a surfactant) and Kolliphor ${ }^{\circledR}$ P 188 (P 188 as a co-surfactant), were prepared in a range of geometrical shapes. The selected geometrical shapes were cylindrical, prism, cube and torus (as shown in Figure 1), providing altered surface area to volume (SA/V) ratios. Fenofibrate (FEN) and cinnarizine (CINN) were selected as model drugs due to their poor water solubilities and their wide use as lipophilic compounds in various studies with lipid-based formulations (12, 54-62). The physicochemical properties and chemical structures of the model drugs are presented in Table 1. The formulations were characterised by differential scanning calorimetry (DSC), wide-angle X-ray scattering (WAXS) and scanning electron microscopy (SEM), and the concentration of the model drugs in the printed S-SMEDDS formulations was quantified using ultra highperformance liquid chromatography (UHPLC). The impact of SA/V ratio on the kinetics of dispersion was studied under simulated gastric and fasted intestinal conditions, and the kinetics of digestion was assessed using a dynamic in vitro lipolysis model under fasted intestinal condition.
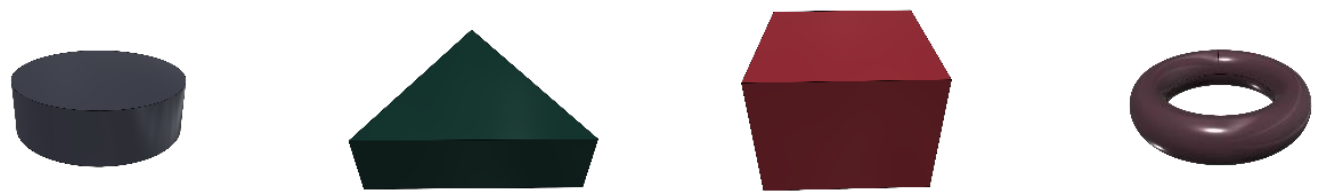

Figure 1. 3D representation of selected geometries of 3DP S-SMEDDS formulations (from left to right - cylindrical, prism, cube and torus). The objects were drawn using AutoDesk 123D Design software (Autodesk Inc. USA) for printing as described. 
Table 1. Physicochemical properties of the model drugs.

\section{Melting Partition}

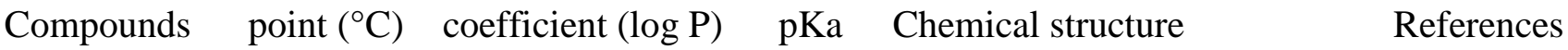

Fenofibrate

(FEN)

N/A

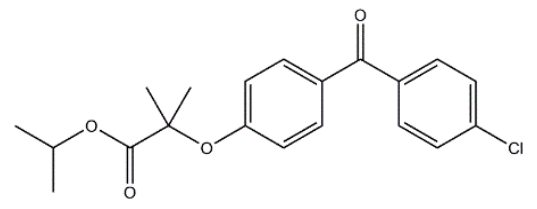

Cinnarizine

1.95

$(\mathrm{CINN})$

$117-120$

5.80

7.51

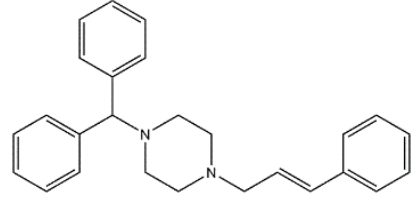

$(65,66)$

\section{Materials and Methods}

\section{Materials and Reagents}

Fenofibrate $(\geq 99 \%)$ and cinnarizine $(\geq 99 \%)$ were purchased from Sigma-Aldrich (Gillingham, Kent, UK). Gelucire ${ }^{\circledR}$ 44/14 (a mixture of C8-C18 mono, di and triacylglycerols and C8-C18 mono and diesters of polyethylene glycol (PEG) and free PEG 32) and Gelucire ${ }^{\circledR}$ 48/16 (PEG 32 stearic esters - a mixture of stearic (C18) and palmitic (C16) acid mono- and diesters of PEG 32) were kindly donated by Gattefossé (Saint-Priest, Lyon, France). Kolliphor ${ }^{\circledR}$ P 188 (Poly(ethylene glycol)-block-poly(propylene glycol)-block-poly(ethylene glycol)) was purchased from BASF (Ludwigshafen, Germany). Trizma ${ }^{\circledR}$ maleate (reagent grade) and bile salt (sodium taurodeoxycholate $(\geq 95 \%)($ NaTDC)) were sourced from Sigma-Aldrich (St. Louis, Missouri). Calcium chloride dihydrate $(\geq 99 \%)$, sodium hydroxide $(\geq 97 \%)$ and ammonium dihydrogen orthophosphate $(\geq 99 \%)$ were obtained from Ajax Finechem Pty. Ltd. (Seven Hills, New South Wales, Australia). Sodium azide, methanol (HPLC grade) and acetonitrile (HPLC grade) were acquired from Merck Schuchardt OHG (Darmstadt, Germany). Phospholipid (1, 2-dioleoyl-sn-glycero-3-phosphocholine (DOPC)) and porcine pancreatic extract were purchased from Cayman Chemical Supply (Sapphire Bioscience, New South Wales, Australia) and Southern Biologicals (Nunawading, Victoria, Australia), respectively. Sodium chloride $(\geq 99 \%$ ) was obtained from Chem-Supply (Gillman, South Australia, Australia) and an analytical grade hydrochloric acid (36\% assay) was purchased from Biolab Pty. Ltd. (Clayton, Victoria, Australia). Water was sourced from a Millipore water purification system using a Quantum ${ }^{\mathrm{TM}}$ EX Ultrapure Organex cartridge (Millipore, Sydney, Australia). 


\section{Preparation of 3DP Drug-loaded S-SMEDDS Formulations in Various}

\section{Geometrical Shapes}

The graphical illustration of the preparation process of 3DP drug-loaded S-SMEDDS formulations is depicted in Figure 2. Briefly, the pre-selected ratios of model drugs, lipid and surfactants (presented in Table 2) were mixed in a glass beaker and placed on the heating plate. The mixtures were heated up to the melting point of the mixtures $\left(80^{\circ} \mathrm{C}\right.$ for FEN-loaded SSMEDDS and $120^{\circ} \mathrm{C}$ for CINN-loaded S-SMEDDS) under magnetic stirring conditions for 30 min to ensure complete mixing of the components and to form solutions of surfactants, lipid and drug. Then, the liquid mixtures were transferred to a KRA-15 extruder stainless steel syringe (Hyrel 3D, Norcross, GA) and allowed to solidify at room temperature. After 15 min, the syringe temperature was increased to $65^{\circ} \mathrm{C}$ to form a dense liquid solution. The temperature was regularly monitored to avoid the solidification or dripping from the syringe nozzle. After 10 min of thermal equilibrium, the mixtures were printed into Figure 1 illustrated geometrical shapes using a Hyrel System 30M syringe-based extrusion 3D printer via $1.5 \mathrm{~mm}$ syringe nozzle (Hyrel 3D, Norcross, GA). The ice-water $\left(<5^{\circ} \mathrm{C}\right)$ was circulated through a modified build plate using a peristaltic pump to create a cold environment for rapid solidification of the printed layers.

Table 2. Selected compositions of prepared S-SMEDDS formulations.

\begin{tabular}{lll}
\hline Substances $(\%)$ & FEN:GEL & CINN:GEL \\
\hline FEN & 7.0 & - \\
CINN & - & 7.0 \\
GEL 44 & 46.5 & 46.5 \\
GEL 48 & 23.3 & 23.3 \\
P 188 & 23.2 & 23.2 \\
Total & 100.0 & 100.0 \\
\hline
\end{tabular}

Values are expressed in $\% \mathrm{w} / \mathrm{w}$. All selected formulations contained 1:1 w/w lipid to surfactants ratio and $0.15: 1.00 \mathrm{w} / \mathrm{w}$ drug to lipid ratio. The above ratios were selected to achieve $1 \mathrm{mg} / \mathrm{mL}$ of drug concentration for $100 \mathrm{mg}$ of lipids in $15 \mathrm{~mL}$ of digestion medium during in vitro lipolysis.

The objects were designed using AutoDesk (AutoDesk Inc. USA) computer-aided design (CAD) software. The CAD files were exported as a stereolithography (.STL) which were 
further converted into Gcode file using Repetrel software (Hyrel 3D, Norcross, GA). The printing parameters determined through prior optimization were as follows: printing nozzle temperature set at $65^{\circ} \mathrm{C}$, nozzle diameter $(1.5 \mathrm{~mm})$, nozzle traveling speed while printing (3 $\mathrm{mm} / \mathrm{s}$ ), layer height $0.4 \mathrm{~mm}$, number of shells (2), nozzle traveling speed while not printing (20 $\mathrm{mm} / \mathrm{s})$, cooling fan speed (25\%), feed rate (1) and material flow at piston drive speed (1100 pulses/s). The infill density was set at 35\% (for the Hyrel System 30M, the recommended infill density is $35 \%$ and the maximum percentage is around $50 \%$ or $60 \%$ ) and infill pattern was selected as concentric. The dimensions of the printed objects were scaled in order to achieve constant weight $(215 \mathrm{mg}$ ) of the 3DP S-SMEDDS formulations. The physical dimensions (length, width and height) of the printed S-SMEDDS formulations were measured using a digital caliper and the average of three measurements is presented in Table 3 along with printing parameters (i.e. number of printed layers and printing time).

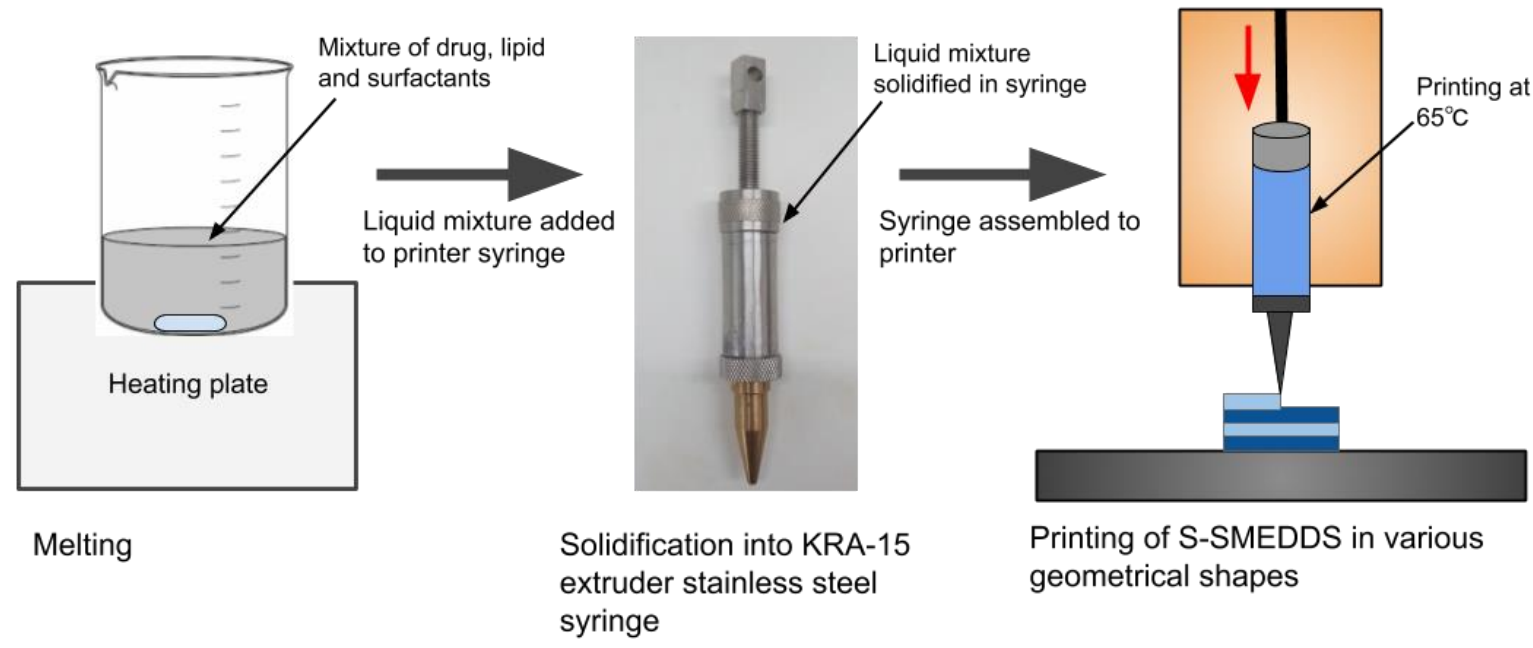

Figure 2. Schematic illustration of the preparation process of 3DP drug-loaded S-SMEDDS formulations.

The control cube S-SMEDDS formulations were prepared manually from a bulk preparation as described previously $(2,12)$. This briefly entailed drawing the molten solution of drug, lipids and surfactant into a disposable syringe, solidifying at $4^{\circ} \mathrm{C}$ in the refrigerator for $24 \mathrm{~h}$, ejecting the solid rod formed and cutting the cube from the rod to desired dimensions using a scalpel. The prepared S-SMEDDS formulations were stored at room temperature until further use. The 
length, width and height of the prepared control cube S-SMEDDS formulations are presented in Table 3 along with 3DP S-SMEDDS formulations.

\section{Characterisation of the 'As-Prepared' 3DP Drug-loaded S-SMEDDS}

\section{Formulations}

The solid bulk materials and prepared drug-loaded S-SMEDDS formulations were characterised for thermal behavior using differential scanning calorimetry (DSC). DSC measurements were performed with DSC 8500 (PerkinElmer, Japan) at $5^{\circ} \mathrm{C} / \mathrm{min}$ heating rate. Samples (average weight of 3-5 mg) were placed into $50 \mu \mathrm{L}$ aluminum pans and sealed with a crimped lid. Nitrogen gas was used as purge gas with a flow rate of approximately $20 \mathrm{~mL} / \mathrm{min}$ for all the experiments. Bulk lipid and surfactants were scanned from 0 to $70^{\circ} \mathrm{C}$ whereas bulk drugs and drug-loaded S-SMEDDS formulations were scanned from 0 to $130^{\circ} \mathrm{C}$. All melting temperatures are stated as extrapolated onset values unless specified otherwise.

The solid state of the bulk drugs and drug-loaded S-SMEDDS formulations was determined using wide-angle X-ray scattering (WAXS) at the SAXS/WAXS beamline at the Australian Synchrotron, ANSTO (67). Similar to the previously reported method, the solid powders or geometrical shapes, covered with Kapton tape, were placed in the X-ray beam and diffractograms of bulk drug powders were acquired at an X-ray beam wavelength of $1.127 \AA$ (energy $11 \mathrm{keV}$ ) and the sample detector distance of $574 \mathrm{~mm}$ (covered the $q$-range of $0.03<q$ $<1.95 \AA^{-1}$ ). The diffractograms of drug-loaded S-SMEDDS formulations were collected at an X-ray beam wavelength of $0.954 \AA$ (energy $13 \mathrm{keV}$ ) and the sample detector distance of 557 mm provided the $q$-range of $0.04<q<1.98 \AA^{-1}$. The three scattering profiles were acquired for $5 \mathrm{~s}$ at every $20 \mathrm{~s}$. The 2D scattering patterns were acquired using Pilatus $1 \mathrm{M}$ detector over an active area of $169 \times 179 \mathrm{~mm}^{2}$. The ScatterBrain Analysis software was used to normalise the data in one-dimension and scattering patterns were converted to intensity and scattering vector $(q)$ to produce a plot of intensity versus scattering vector $(q)$, using the equation $q=$ $(4 \pi / \lambda) \sin (\theta / 2)$, where ' $\lambda$ ' is the wavelength and ' $\theta$ ' is the scattering angle. The average of three scattering patterns of Kapton tape was collected separately and subtracted as background from all scattering profiles. The diffractograms of 3DP S-SMEDDS formulations were collected for the cylindrical shapes only. For a direct comparison, the scattering profiles of the drug-free SSMEDDS formulations were also acquired to confirm the possible overlapping of the peaks 
from the formulation components with drug peaks (data presented as Figure S1 in the Electronic Supporting Information).

The morphology and porosity of 3DP S-SMEDDS formulations were characterised using scanning electron microscopy (SEM). The electron microscopy images of the surface (edge of the geometrical shapes) and cross-sections were acquired using a benchtop SEM (Phenom. FEI, Dillenburgstraat 9T, Netherlands). Prior to SEM analysis, the samples were placed on doublesided adhesive carbon glue and coated with gold using sputter coater (Emitech K550x, Kent, UK).

Furthermore, the content of the model drugs in the 3DP S-SMEDDS formulations was determined using ultra high performance liquid chromatography (UHPLC). Approximately 50 $\mathrm{mg}$ of the drug-loaded S-SMEDDS formulations (cylindrical shapes) was dissolved into 100 $\mathrm{mL}$ of respective mobile phases at $37^{\circ} \mathrm{C}$ with magnetic stirring to form a clear transparent solution. The acquired solutions were further diluted with respective mobile phases until the concentration of the model drugs fell within the range of the standard curve.

A previously reported HPLC method was followed to determine the concentration of FEN and CINN in the 3DP S-SMEDDS formulations (12). The Nexera X2 UHPLC system comprised of a Shimadzu DGU-20A system controller, LC-30AD solvent delivery module, SIL-30AC autosampler and CTO-20AC column oven model at $40^{\circ} \mathrm{C}$, attached to the SPD-M30A UV detector (Shimadzu Corporation, Kyoto, Japan) and RF-20AXS fluorescence detector (Shimadzu Corporation, Kyoto, Japan). A reverse-phase C18 Waters Symmetry ${ }^{\circledR}$ column (4.6 $\mathrm{mm} \times 75 \mathrm{~mm}, 3.5 \mu \mathrm{m}$, MA, the USA) was used.

For FEN, the mobile phase for an isocratic premix was composed of acetonitrile and Milli-Q water (80:20) with $0.1 \%(\mathrm{v} / \mathrm{v})$ formic acid. The analysis was performed at the flow rate of 1 $\mathrm{mL} / \mathrm{min}$ at the injection volume of $20 \mu \mathrm{L}$ and the drug was detected at $286 \mathrm{~nm} \mathrm{UV}$ wavelength. For CINN, the mobile phase for an isocratic premix was composed of acetonitrile and Milli-Q water (50:50) with ammonium dihydrogen orthophosphate at $20 \mathrm{mM}$ where $\mathrm{pH}$ was adjusted to 4.2. The analysis was performed at the flow rate of $1 \mathrm{~mL} / \mathrm{min}$ at the injection volume of 20 $\mu \mathrm{L}$ and the drug was detected using fluorescence detector at a wavelength of Excitation 249 $\mathrm{nm}$ and Emission $311 \mathrm{~nm}$. The study was performed in triplicate using different section of 3DP dose forms and the concentration of drugs was determined by comparison to the standard curve. 


\section{Characterisation of Dispersion and Digestion Behaviour of Drug-loaded S- SMEDDS Formulations}

The dispersion study was performed to assess the impact of SA/V ratio on the dispersion process of S-SMEDDS formulations. The study was performed using the paddle apparatus USP type II (Erweka DT6, Heusenstamm, Germany) in $400 \mathrm{~mL}$ of simulated gastric medium (0.1 $\mathrm{M} \mathrm{HCl}$ ) at $37^{\circ} \mathrm{C}$ and at $100 \mathrm{rpm}$ paddle speed. The two solid dosage forms (single dose weight of approximately $215 \mathrm{mg}$ ) were added into the dispersion medium and the time required for the formulations to spontaneously self-emulsify and form an emulsion was visually determined. The dispersion time was noted at the time at which the visible solid dosage forms were completely dispersed to the eye. The study was performed in triplicate.

The fasted micellar media used for digestion experiments, reflecting the fasted state of the human intestinal condition, was prepared according to a previously reported protocol using digestion buffer and used as digestion medium $(2,12)$. The standard digestion buffer of $\mathrm{pH}$ $6.500 \pm 0.003$ solutions containing $50 \mathrm{mM}$ Trizma $^{\circledR}$ maleate, $150 \mathrm{mM} \mathrm{NaCl}, 5 \mathrm{mM} \mathrm{CaCl} 2 . \mathrm{H}_{2} \mathrm{O}$ and $6 \mathrm{mM} \mathrm{NaN}_{3}$, was prepared where $\mathrm{pH}$ was adjusted to $6.500 \pm 0.003$ using $\mathrm{HCl}$ and $\mathrm{NaOH}$ solutions. The fasted state micellar solution was prepared by adding bile salts and phospholipids at concentrations of $5 \mathrm{mM}: 1.25 \mathrm{mM}$, respectively, into the digestion buffer. Briefly, the required quantity of DOPC (phospholipid) was dissolved into 1-2 mL chloroform into a round bottom flask. A thin-film of DOPC at the bottom of the flask was achieved by evaporating chloroform using a rotary evaporator (BÜCHI, Rotavapor-RE, Switzerland) under vacuum conditions. The required quantity of digestion buffer and NaTDC (bile salt) were added into the round bottom flask and the mixture was sonicated in a sonicating bath at room temperature for approximately $1 \mathrm{~h}$ to achieve a clear micellar solution. The prepared micellar solution was stored at $4{ }^{\circ} \mathrm{C}$ in the refrigerator and used within two weeks.

The freeze-dried pancreatic lipase was also prepared in accordance with a previously reported protocol at a concentration of approximately $1000 \mathrm{TBU}$ (tributyrin units)/mL of the digest where lipase activity was determined by a separate tributyrin test (2). For each digestion experiment, the freeze-dried lipase was reconstituted at approximately $286 \mathrm{mg} / \mathrm{mL}$ of digestion buffer, such that $1.5 \mathrm{~mL}$ of solution added to $13.5 \mathrm{~mL}$ of the digest (see below) provided the required activity.

In vitro lipolysis experiments were performed to investigate the effect of $\mathrm{SA} / \mathrm{V}$ ratio values on the kinetics of digestion at fasted intestinal condition $(2,58,68,69)$. The model consisted of a 
pH-stat apparatus (Metrohm ${ }^{\circledR}$, Switzerland), Titrando 802 propeller stirrer, a glass $\mathrm{pH}$ electrode (iUnitrode), 800 Dosino dosing unit attached to a $5 \mathrm{~mL}$ autoburette (Metrohm ${ }^{\circledR}$, Switzerland) and a thermostatted glass vessel at $37^{\circ} \mathrm{C}$ with stirred conditions was operated using Tiamo 2.0 software. For each digestion experiment, $13.5 \mathrm{~mL}$ of the digestion medium (fasted intestinal micelles) was added into a thermostatted glass vessel where the $\mathrm{pH}$ was readjusted to $6.500 \pm$ 0.003 using $\mathrm{NaOH}$ and $\mathrm{HCl}$ solutions. After 5 min of thermal equilibrium, a single drug-loaded geometrical shape (approximately $215 \mathrm{mg}$ ) and $1.5 \mathrm{~mL}$ of reconstituted pancreatic lipase were added to the glass vessel at the same time to investigate the impact of geometry on dispersion and digestion kinetics. During lipolysis, the $\mathrm{pH}$ of the digestion medium dropped due to the liberation of ionised-fatty acids from S-SMEDDS formulations. Therefore, 0.6 M NaOH solution was titrated via auto-burette to maintain the $\mathrm{pH}$ at a pre-selected value of $6.500 \pm 0.003$. The duration of all lipolysis experiments was $60 \mathrm{~min}$ and the extent of enzymatic digestion was reflected in the consumed volume of $\mathrm{NaOH}$ to neutralize the liberated fatty acids during digestion. The percentage of ionised fatty acids was calculated using the liberated moles of fatty acids relative to the theoretical moles of fatty acids equivalent in the formulation. In the current study, the results are presented for ionised-fatty acids only to quantify the effect of geometry on the digestion for initial $30 \mathrm{~min}$ until the solid dosage form completely dispersed into the digestion medium and formed an emulsion.

\section{Statistical analysis}

For all performed experiments, the obtained results are expressed as mean \pm standard deviation and were replicated at least three times. The results of dispersion and digestions were analysed using the IBM SPSS Statistics 25 and a one-way variance test (ANOVA) with Tukey post hoc analysis was performed. The $p$-values are expressed by $(p$-value $<0.05)$ or $(p$-value $>0.05)$ at the point of comparison.

\section{Results and Discussion}

\section{Physical Parameters and Printing of S-SMEDDS Formulations}

Firstly, this proof of concept study proved that it was feasible to prepare drug-loaded SSMEDDS formulations without a solid-phase carrier using 3DP technology. As shown in Figure 3, the printed S-SMEDDS were intact, well-defined and deemed acceptable for normal 
handling. However, the formal pharmacopeia testing would be necessary to confirm the strength and friability of the prepared dose forms during formal product development. Although the minor slumping was evident and the significant differences between the shapes was apparent. This is the first time that a level of control over geometry of S-SMEDDS dosage forms has been demonstrated. During printing, the syringe temperature (configured to $65^{\circ} \mathrm{C}$ ) was carefully controlled to avoid solidification of the material in the nozzle tip at a lower temperature or dripping at a higher temperature. The circulation of ice-water $\left(<5^{\circ} \mathrm{C}\right)$ through the modified build plate stimulated the solidification of printed layers and enabled the binding of layers, offering a single solid object. The use of a Peltier cooled plate would be more preferable in future studies with such systems, but the water cooled build plate sufficed for this proof of concept.

The drug-loading percentage was consistent $(7 \% \mathrm{w} / \mathrm{w})$ for both model drugs, with the recovered concentration of the model drugs in the 3DP S-SMEDDS formulations being within a small percentage variation of the theoretical loading (data presented as Figure S2 in the Electronic Supporting Information) precluding settling or segregation in the printer syringe. The presence of the drugs did not exert any effect on the printing process. However, as highlighted in Table 3, a slight adjustment in the objects' dimensions were required to achieve consistent weight of $215 \mathrm{mg}$, indicating slight differences in the density of the printed liquid. During printing, no solid undissolved particles of the drugs were observed and the prepared formulations were transparent straw coloured liquid of drugs dissolved in lipid and surfactant without undissolved solid thus it was anticipated that the drug particles were still dissolved into the molten lipid-surfactant matrix at printing temperature $\left(65^{\circ} \mathrm{C}\right)$.

Table 3. Physical dimensions and printing parameters of 3DP FEN-loaded S-SMEDDS (FEN S-SMEDDS), CINN-loaded S-SMEDDS (CINN S-SMEDDS) and physical dimensions of the control cube S-SMEDDS formulations $(n=3)$.

\begin{tabular}{llll}
\hline Shapes & Length x width x height $(\mathrm{mm})$ & Printed layers & Printing time (min) \\
\hline$\underline{\text { FEN S-SMEDDS }}$ & & & \\
Cylindrical & $11 \times 11 \times 3.3$ & 9 & 3.2 \\
Prism & $12.3 \times 12.3 \times 3.8$ & 11 & 3.3 \\
Cube & $7.3 \times 7.3 \times 5.5$ & 15 & 3.1 \\
Torus & $13.7 \times 13.7 \times 3.4$ & 9 & 3.1 \\
Control cube & $6.1 \times 6.4 \times 5.3$ & - & - \\
\hline
\end{tabular}




\section{CINN S-SMEDDS}

$\begin{array}{llll}\text { Cylindrical } & 9.1 \times 9.1 \times 2.5 & 8 & 2.2 \\ \text { Prism } & 12 \times 12 \times 3.5 & 9 & 2.3 \\ \text { Cube } & 7 \times 7 \times 4.5 & 12 & 2.3 \\ \text { Torus } & 12.3 \times 12.3 \times 3.4 & 9 & 2.4 \\ \text { Control cube } & 6.1 \times 6.3 \times 5.1 & - & -\end{array}$

The printed objects in four geometrical shapes (three compact geometries with different edges and surfaces and one with a hole in the middle) with different SA/V ratios and the prepared control cube are illustrated in Figure 3 and their physical parameters are tabulated in Table 4 and 5. All 3DP S-SMEDDS formulations were printed at a constant weight of approximately $215 \mathrm{mg}$ (containing $15 \mathrm{mg}$ FEN or CINN) by adjusting the tablet XYZ dimensions, aimed at maintaining the same drug content in each dosage form.

For both drug-loaded S-SMEDDS formulations, the torus shape provided the largest SA/V ratio values due to the hole in the middle compared to compact geometries and the differences in the SA/V ratio values for compact geometries were relatively small (Table 4 and 5). Although, the SA/V ratio values for FEN-loaded S-SMEDDS formulations were larger compared to CINN-loaded S-SMEDDS formulations due to smaller XYZ dimensions and extrusion of the apparently slightly denser solution.

The printed geometrical shapes in Figure 3 were not perfect in comparison to the intended geometries, but very close. For instance, the cylindrical and cube shapes looked similar from the top view for both drug-loaded S-SMEDDS formulations. This phenomenon can be explained by the low-resolution of the nozzle diameter and the less-controlled spatial distribution of the printed layers at the liquid state. This can be further improved by enhancing the resolution (by using smaller nozzle diameter) and printing mixtures in a semi-solid state. The slight distortion of the solid dosage forms made it challenging to calculate the accurate physical dimensions of the formulations thus the physical dimension presented in Tables 4 and 5 were estimated from the mean value of the measurements of the physical dimensions of the objects. 

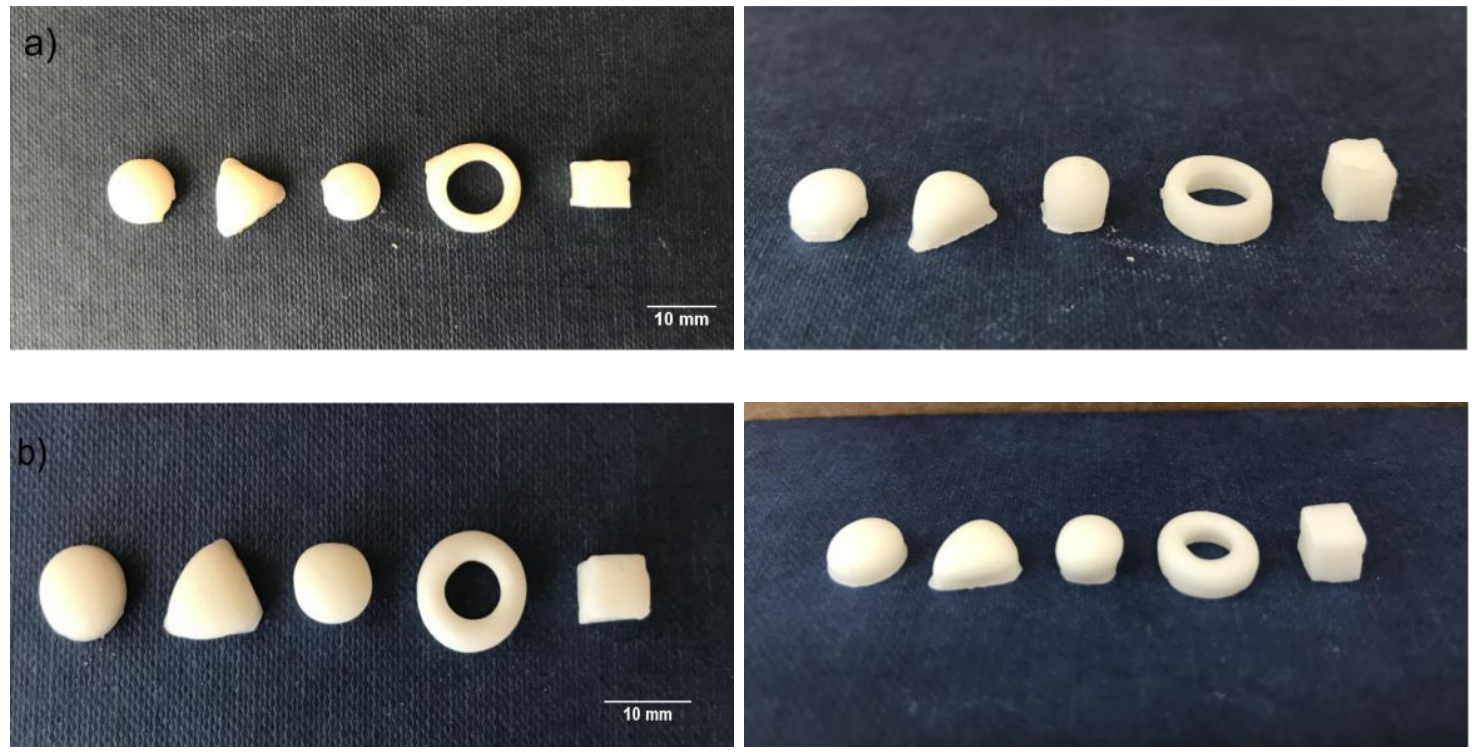

Figure 3. Images of drug-loaded S-SMEDDS formulations in various geometrical shapes (from left to right - cylindrical, prism, cube, torus and control cube) of (a) FEN-loaded (top view and side view) and (b) CINN-loaded (top view and side view), S-SMEDDS formulations. The cylindrical and cube shapes looked similar at least from top view, due to low-resolution of the nozzle diameter and the less-controlled spatial distribution of the printed layers at the liquid state.

Table 4. Physical parameters of FEN-loaded S-SMEDDS formulations ( $n=3$, mean \pm SD).

\begin{tabular}{lllll}
\hline Shape & Surface area $\left(\mathrm{mm}^{2}\right)$ & Volume $\left(\mathrm{mm}^{3}\right)$ & SA/V ratio $\left(\mathrm{mm}^{-1}\right)$ & Weight $(\mathrm{mg})$ \\
\hline Cylindrical & $306.0 \pm 3.7$ & $317.8 \pm 5.6$ & $0.963 \pm 0.006$ & $214.0 \pm 3.5$ \\
Prism & $290.3 \pm 1.1$ & $285.4 \pm 2.1$ & $1.017 \pm 0.004$ & $216.0 \pm 7.2$ \\
Cube & $266.4 \pm 1.6$ & $292.2 \pm 2.8$ & $0.912 \pm 0.003$ & $218.0 \pm 1.5$ \\
Torus & $327.6 \pm 4.3$ & $197.9 \pm 5.0$ & $1.655 \pm 0.020$ & $214.0 \pm 7.8$ \\
Control cube & $210.9 \pm 18.7$ & $208.0 \pm 27.4$ & $1.014 \pm 0.044$ & $206.3 \pm 4.7$ \\
\hline
\end{tabular}

Table 5. Physical parameters of CINN-loaded S-SMEDDS formulations ( $n=3$, mean \pm SD).

\begin{tabular}{lllll}
\hline Shape & Surface area $\left(\mathrm{mm}^{2}\right)$ & Volume $\left(\mathrm{mm}^{3}\right)$ & SA/V ratio $\left(\mathrm{mm}^{-1}\right)$ & Weight $(\mathrm{mg})$ \\
\hline Cylindrical & $226.4 \pm 2.6$ & $197.6 \pm 1.9$ & $1.146 \pm 0.011$ & $214.0 \pm 4.6$ \\
Prism & $269.5 \pm 4.4$ & $252.8 \pm 6.1$ & $1.066 \pm 0.009$ & $215.0 \pm 6.2$ \\
Cube & $222.5 \pm 1.6$ & $218.4 \pm 1.9$ & $1.019 \pm 0.003$ & $213.0 \pm 9.8$ \\
\hline
\end{tabular}




\begin{tabular}{lllll}
\hline Torus & $347.6 \pm 7.9$ & $248.9 \pm 8.3$ & $1.397 \pm 0.032$ & $218.0 \pm 4.0$ \\
Control cube & $211.7 \pm 17.4$ & $208.4 \pm 19.3$ & $1.016 \pm 0.068$ & $218.3 \pm 5.7$ \\
\hline
\end{tabular}

DSC and X-ray scattering at wide-angle (WAXS) were employed to investigate the physical state of the model drugs in the prepared S-SMEDDS formulations. As shown in Figure 4, the bulk components expressed endothermic peaks corresponding to the melting points of the individual components. The bulk drugs also presented drug endotherms representing melting of the crystalline raw drug materials (Figure 4a). Both of the drug-loaded formulations exhibited only an endotherm at approximately $44-48^{\circ} \mathrm{C}$ that correlates to the melting of the excipients and the drug endotherms were absent (Figure 4b). This indicates a significant physicochemical interaction between the components in the mixture formulations and suggest that the drugs were either in amorphous state or molecularly dispersed into the molten lipidsurfactants matrix at an increased temperature. Additionally, it also indicates that the drugs have dissolved into the lipid formulations at the printing temperature $\left(65^{\circ} \mathrm{C}\right)$ consistent with the visual observations.
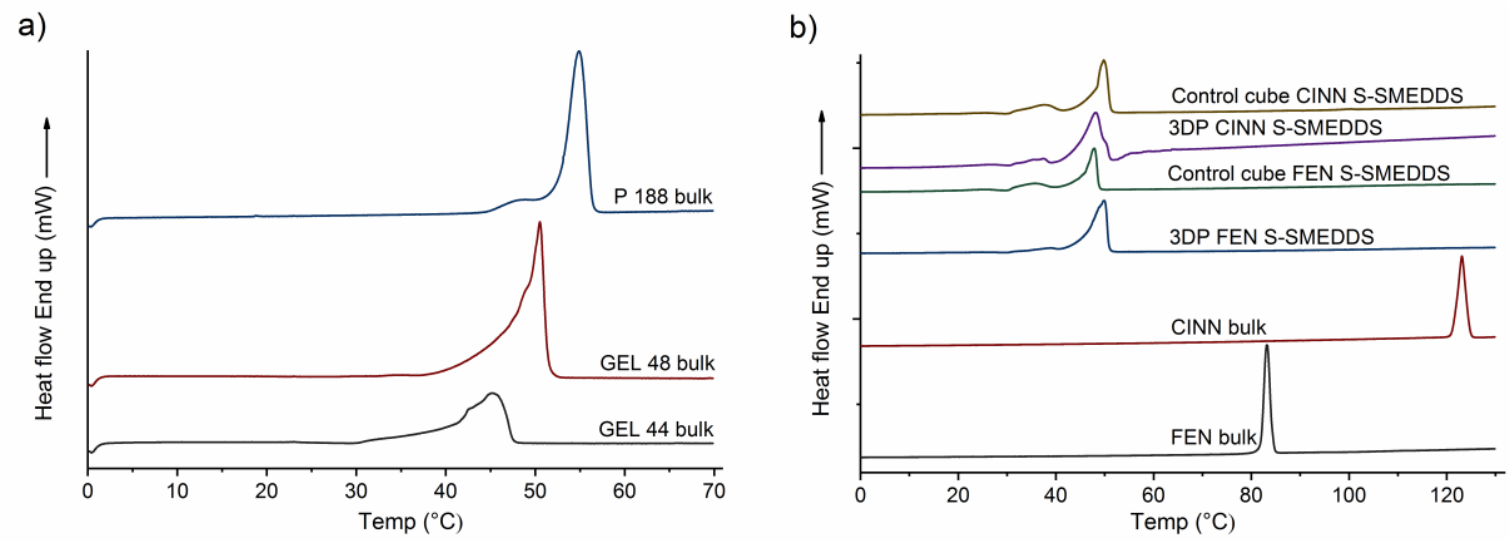

Figure 4. DSC thermal traces of (a) bulk GEL 44, bulk GEL 48 and bulk P 188, and (b) bulk FEN, bulk CINN, 3DP FEN-loaded S-SMEDDS, control cube FEN-loaded S-SMEDDS, 3DP CINN-loaded S-SMEDDS, and control cube CINN-loaded S-SMEDDS formulations.

The scattering patterns of bulk model drugs and drug-loaded formulations are presented in Figure 5. Additionally, the scattering profiles of drug-free S-SMEDDS formulations were compared to drug-loaded S-SMEDDS formulations (data presented as Figure $\mathrm{S} 1$ in the 
Electronic Supporting Information) and confirmed that there were no overlapping of the peaks between the excipients and the drug. The bulk FEN and bulk CINN displayed crystalline state by presenting FEN major diffraction peaks at $q$ of $0.84,1.02,1.12,1.14$ and $1.18 \AA^{-1}$ (Figure 5a) and CINN diffraction peaks at $q$ of $0.72,1.09$ and $1.32 \AA^{-1}$ (Figure 5b). Consistent with previous observation for similar type of formulations, the drugs corresponding peaks were evident with similar $q$-range (with additional peaks from other components of the formulations (confirmed from Figure S1)) but lower intensities than the bulk form (12), indicating the drugs partially retained its crystallinity after mixing. The results clearly suggested that the drugs were not in the amorphous state in the final mixture formulations and indicated that the drugs were most likely solubilised into the molten lipid-surfactants matrix at high temperature but recrystallised upon cooling. The slight difference in the $q$-values of the drug diffractions peaks for drug-loaded formulations compared to bulk samples was most possibly due to the difference in the experimental setup and some orientation of crystalline material in the reference diffraction patterns.
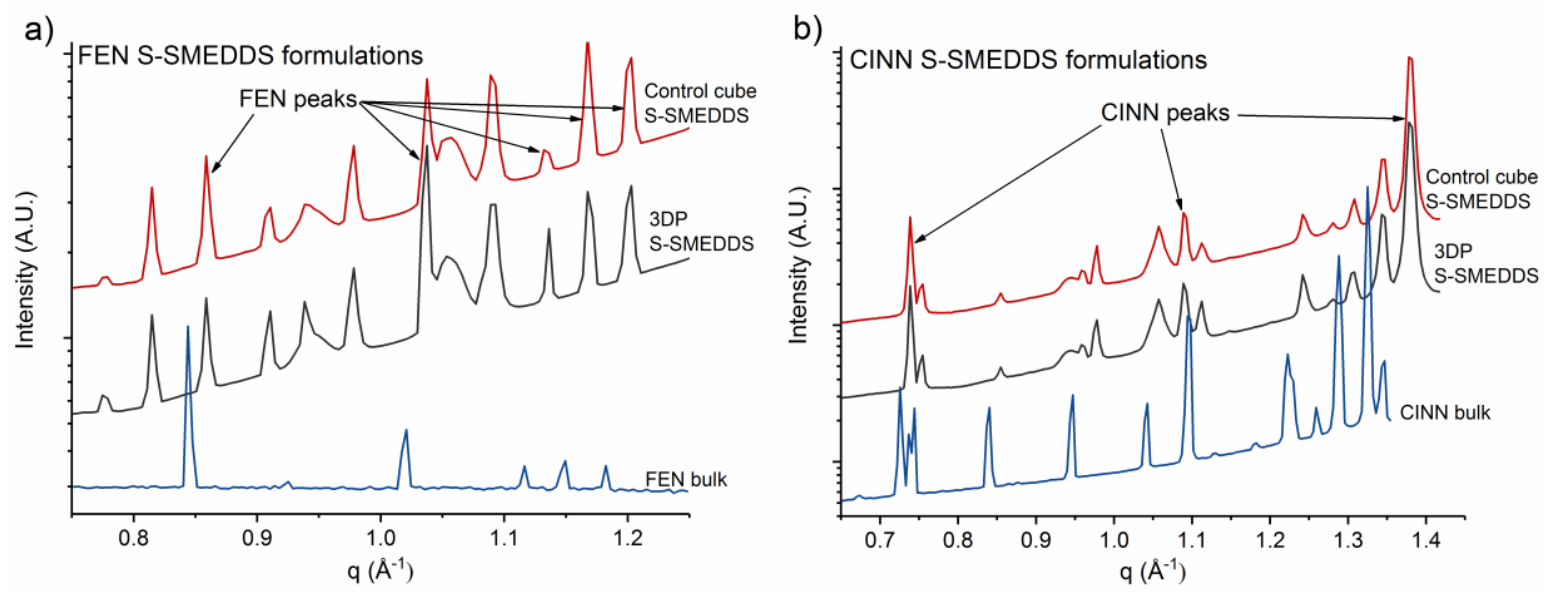

Figure 5. The wide-angle X-ray scattering (WAXS) profiles of (a) bulk FEN, 3DP and control cube FEN-loaded S-SMEDDS and (b) bulk CINN, 3DP and control cube CINN-loaded SSMEDDS, formulations collected at Australian Synchrotron, ANSTO using the SAXS/WAXS beamline. FEN bulk and CINN bulk scattering patterns were arbitrarily scaled for the graphing purpose. 


\section{Morphology of the 3D Printed Solid Object}

SEM was employed to analyse the morphology and porosity of drug-loaded 3DP S-SMEDDS

formulations. In Figure 6, the electron microscope images indicated the presence of crystalline FEN particles within the printed formulations with large solid particles being apparent (highlighted with a circle). The images also clearly showed the deposition of individual layers connected to each other (Figure 6). The layer diameter $\sim 400-600 \mu \mathrm{m}$ was consistent with the nozzle diameter (theoretically $0.4 \mathrm{~mm}$ although this was not specifically measured). Most possibly, the extrusion of liquid solution affected the layer deposition process and led to slight curl of the liquid materials on the build plate and/or previous printed layers, providing uneven layer thickness. This can be further improved by enhancing the resolution (by using smaller nozzle diameter) and printing mixture at semi-solid state or with further additives to control rheology. However, as shown in Figure 6, the printed layers still fused together very well, leading to the strength of the 3DP dosage forms. While the materials printed in this study formed an essentially solid low porosity object, future studies will include using the advantage of 3DP in building in increasingly porous structure to provide even further control over dispersion behaviour.
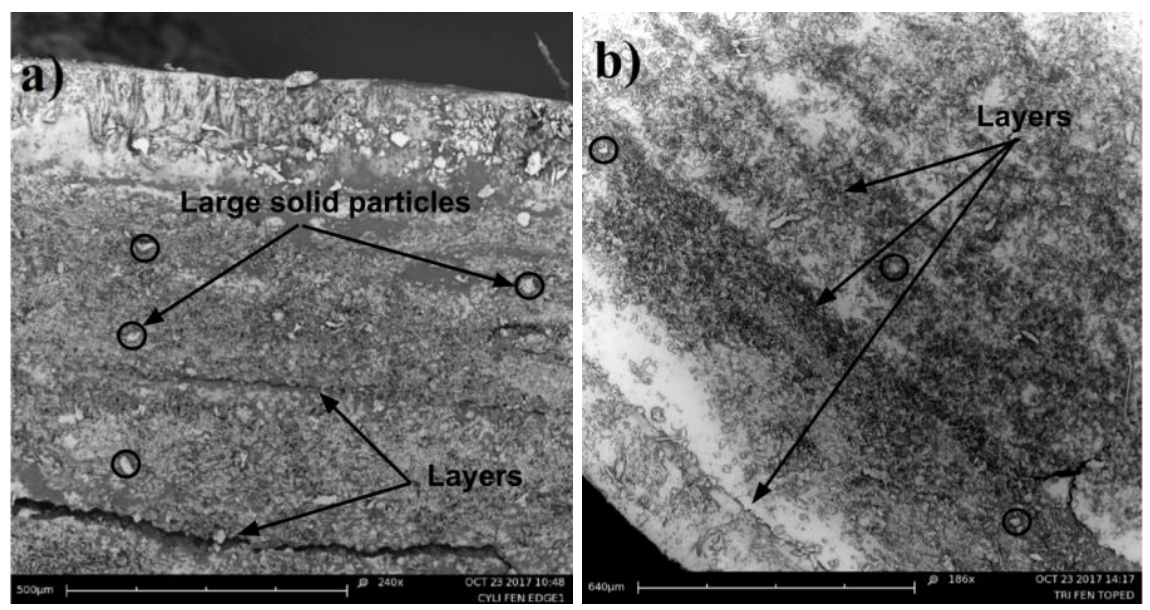

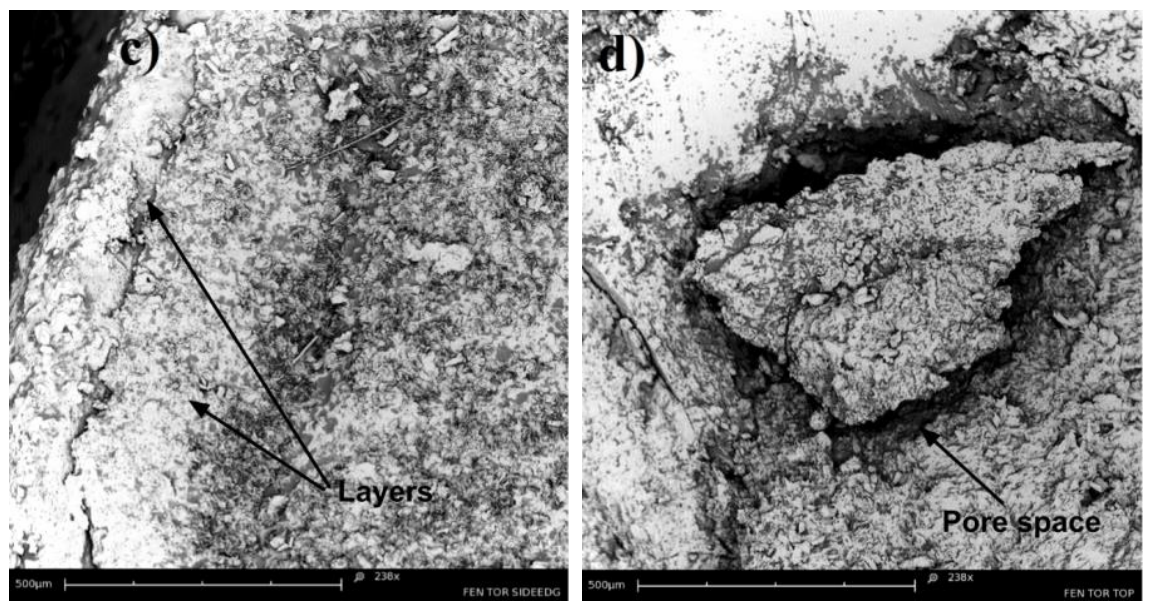

Figure 6. SEM images of 3DP dosage forms of (a) cylindrical surface edge (b) prism surface edge (c) torus surface edge and (d) torus cross-section, of FEN-loaded S-SMEDDS formulations. The images clearly demonstrated the individual layers, large particles and pore space where the layers fused together and formed a solid object. The scale bar in Panels (a), (c) and (d) is $500 \mu \mathrm{m}$, whereas in Panel (b) it is $640 \mu \mathrm{m}$.

\section{Correlation of Dispersion and Digestion Kinetics of Drug-loaded S-} SMEDDS Formulations with SA/V Ratio Values

The correlation of SA/V ratio values to the dispersion time of drug-loaded S-SMEDDS formulations under simulated gastric condition is presented in Figure 7. The dispersion time was noted when the solid dosage forms completely dispersed to the naked eye and are tabulated in Table S1 in the Electronic Supporting Information.

The results clearly indicated that geometry plays a pivotal role in defining the dispersion process. For both drug-loaded systems, the order of dispersion time was (fastest first) torus > prism $>$ control cube $>$ cylindrical and cube $(p<0.05)$. The dispersion time generally trended with changes in the SA/V ratio values with the shortest dispersion time for torus shapes (42 min and 58 min for FEN- and CINN-loaded S-SMEDDS, respectively) and the longest for cube shapes (66 $\mathrm{min}$ and $81 \mathrm{~min}$ for FEN- and CINN-loaded S-SMEDDS, respectively). The magnitude of the difference in dispersion time was higher between torus and other compact geometries whereas the difference was minimal between compact geometries. The dependence on $\mathrm{SA} / \mathrm{V}$ ratio for the compact geometries may have been clearer under alternative dispersion testing conditions (such as a slower paddle speed), nevertheless the higher SA/V ratio 
geometries generally provided the fastest dispersion rate. Overall, the dispersion time of CINNloaded S-SMEDDS formulations was longer compared to FEN-loaded S-SMEDDS formulations likely due to smaller SA/V ratio values and the extrusion of dense layers from the heavier liquid solution.
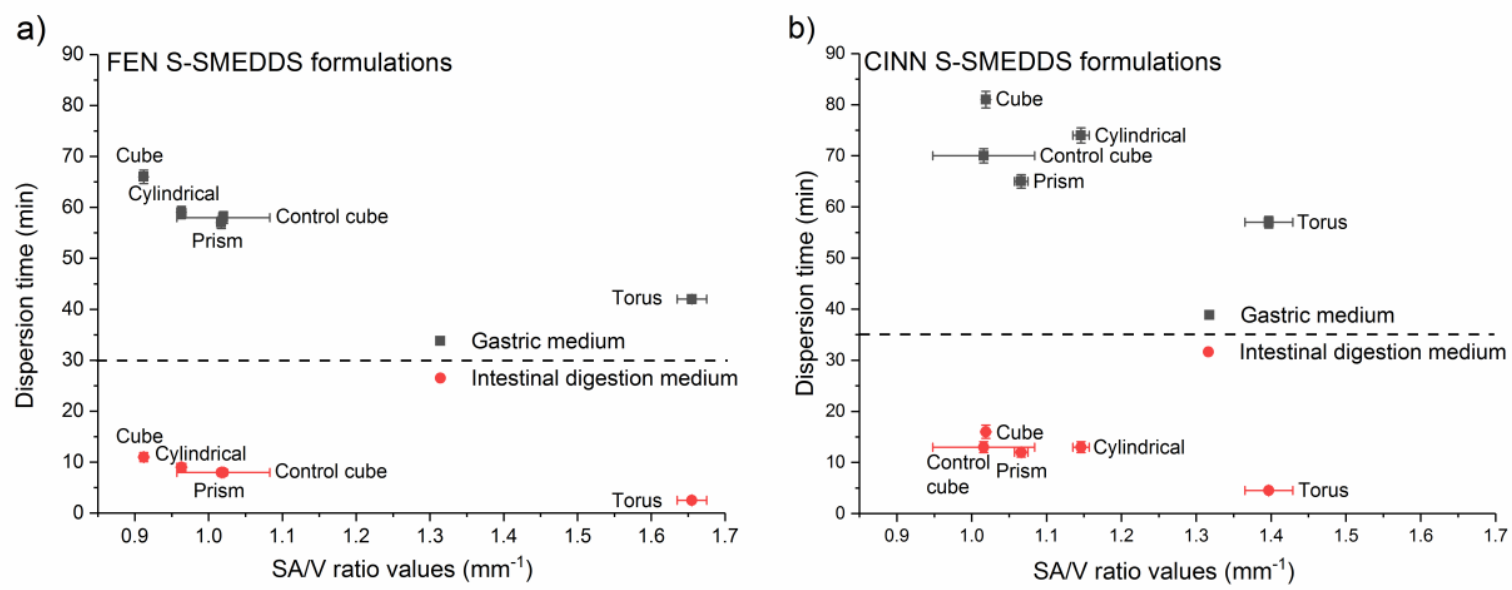

Figure 7. The correlation of dispersion time to $\mathrm{SA} / \mathrm{V}$ ratio values for various geometrical shapes of (a) FEN-loaded S-SMEDDS and (b) CINN-loaded S-SMEDDS, formulations performed at the simulated gastric condition in USP II apparatus and at fasted intestinal digestion condition during digestion in in vitro lipolysis model $(n=3$, mean $\pm \mathrm{SD})$.

Furthermore, the dispersion and digestion of the 3D printed objects were then studied under fasted intestinal lipolysis condition using the previously reported in vitro lipolysis model (70). As seen in Figure 7, the dispersion time of geometrical shapes in the fasted intestinal digestion medium was overall much faster $(p<0.05$ for both drug-loaded S-SMEDDS formulations) than in gastric medium. The dispersion rate followed a similar rank order for the various geometries as that seen in gastric medium, with the shortest dispersion time for the high SA/V ratio value torus shape for both drug-loaded S-SMEDDS formulations. It was anticipated that wetting of the dose form and the penetration of dispersion medium through hydrophilic regions of the printed material was promoted in the presence of endogenous amphiphilic components such as bile salt and phospholipid present in the simulated fasted intestinal medium, which led to faster dispersion process in comparison to simulated gastric condition (Figure 7).

During digestion, for both drug-loaded systems, the lipase had better access to the digestible components of the torus shapes due to higher SA/V ratio in comparison to the compact 
geometries (i.e. cylindrical, prism and cube). Hence, the extent of digestion was higher for torus shapes during the early stage of digestion (see Figure S3 in the Electronic Supporting Information). However, as anticipated, the exposure of digestible components increased for all geometrical shapes after dispersion and thus resulted into similar digestion kinetics and extent of digestion for all geometrical shapes after the initial dispersion phase. This indicated that geometry plays a role in the overall kinetics of digestion only until dispersion of the geometrical shapes has taken place.

\section{Conclusions}

This study demonstrated the concept of using 3DP technology for the preparation of SSMEDDS formulations with controlled three-dimensional geometry without a solid-phase carrier. It was straightforward to prepare and fabricate the dosage forms into various geometrical shapes (cylindrical, prism, torus and cube). This demonstrated the versatility of 3DP as a novel manufacturing process for solid lipid-based formulations. Solid-state analysis revealed the crystalline state of the model drugs in the final dosage forms and microscopic images showed fusion of the printed layers forming a solid object. The dispersion of SSMEDDS formulations was faster in fasted intestinal condition attributed to the presence of bile salts and phospholipids, and the dispersion kinetics of drug-loaded S-SMEDDS formulations showed a clear dependence on the SA/V ratio values, indicating the impact of geometrical shape on dispersion time. This proof of concept study indicates that with optimisation, 3DP can offer an alternative technique to prepare S-SMEDDS dose forms with geometrical flexibility and complexity suitable for the development of personalised lipid-based dosage forms.

\section{Acknowledgments}

The authors would like to thank Gattefossé (Saint-Priest, Lyon, France) for financial support for Kapilkumar's Ph.D. research study. The authors would also like to acknowledge Monash University (Melbourne, Australia) for the financial support for the PharmAlliance Scheme and University College London (London, UK) for allowing the use of a 3D printer for this work. WAXS experiments were undertaken on the SAXS/WAXS beamline at the Australian Synchrotron, ANSTO, Australia. UPLC study was conducted in the HMST Laboratory located 
at Monash University, Parkville. The studies were supported by the Australian Research Council Discovery Grant scheme (DP160102906) and Ben Boyd is funded under ARC Future Fellowship (FT120100697).

\section{Conflict of Interest}

Vincent Jannin is Research Director at Gattefossé (Saint-Priest, Lyon, France), who manufactures the Gelucire ${ }^{\circledR} 44 / 14$ and Gelucire ${ }^{\circledR} 48 / 16$ used in this study.

\section{References}

1. Porter CJ, Trevaskis NL, Charman WN. Lipids and lipid-based formulations: optimizing the oral delivery of lipophilic drugs. Nature Reviews Drug Discovery. 2007;6(3):231.

2. Vithani K, Hawley A, Jannin V, Pouton C, Boyd BJ. Inclusion of Digestible Surfactants in Solid SMEDDS Formulation Removes Lag Time and Influences the Formation of Structured Particles During Digestion. The AAPS journal. 2017:1-11.

3. Pouton CW. Lipid formulations for oral administration of drugs: non-emulsifying, selfemulsifying and 'self-microemulsifying' drug delivery systems. European Journal of Pharmaceutical Sciences. 2000;11, Supplement 2:S93-S98.

4. Oh DH, Kang JH, Kim DW, Lee B-J, Kim JO, Yong CS, Choi H-G. Comparison of solid selfmicroemulsifying drug delivery system (solid SMEDDS) prepared with hydrophilic and hydrophobic solid carrier. International journal of pharmaceutics. 2011;420(2):412-418.

5. Kang BK, Lee JS, Chon SK, Jeong SY, Yuk SH, Khang G, Lee HB, Cho SH. Development of self-microemulsifying drug delivery systems (SMEDDS) for oral bioavailability enhancement of simvastatin in beagle dogs. International journal of pharmaceutics. 2004;274(1):65-73.

6. Holm R, Porter CJ, Edwards GA, Müllertz A, Kristensen HG, Charman WN. Examination of oral absorption and lymphatic transport of halofantrine in a triple-cannulated canine model after administration in self-microemulsifying drug delivery systems (SMEDDS) containing structured triglycerides. European journal of pharmaceutical sciences : official journal of the European Federation for Pharmaceutical Sciences. 2003;20(1):91-97.

7. Jannin V, Chambin O. Self emulsifying drug delivery systems. STP Pharma Techniques Pratiques Reglementations. 2005;15(3):247-254.

8. Meghani Nilesh SD. Self micro-emulsifying drug delivery system (SMEDDS): A promising tool to improve bioavailability. Journal of Pharmacy and Phytotherapeutics. 2013(2(1)):17-21.

9. Fernandez S, Jannin V, Rodier J-D, Ritter N, Mahler B, Carrière F. Comparative study on digestive lipase activities on the self emulsifying excipient Labrasol®, medium chain glycerides and PEG esters. Biochimica et Biophysica Acta (BBA)-Molecular and Cell Biology of Lipids. 2007;1771(5):633-640.

10. Gurram AK, Deshpande PB, Kar SS, Nayak UY, Udupa N, Reddy MS. Role of Components in the Formation of Self-microemulsifying Drug Delivery Systems. Indian Journal of Pharmaceutical Sciences. 2015;77(3):249-257.

11. Pouton CW. Formulation of self-emulsifying drug delivery systems. Advanced drug delivery reviews. 1997;25(1):47-58.

12. Vithani K, Hawley A, Jannin V, Pouton C, Boyd BJ. Solubilisation behaviour of poorly watersoluble drugs during digestion of solid SMEDDS. European Journal of Pharmaceutics and Biopharmaceutics. 2018;130:236-246.

13. Kalepu S, Manthina M, Padavala V. Oral lipid-based drug delivery systems - an overview. Acta Pharmaceutica Sinica B. 2013;3(6):361-372. 
14. Nazzal S, Khan MA. Controlled release of a self-emulsifying formulation from a tablet dosage form: Stability assessment and optimization of some processing parameters. International journal of pharmaceutics. 2006;315(1):110-121.

15. Yi T, Wan J, Xu H, Yang X. A new solid self-microemulsifying formulation prepared by spraydrying to improve the oral bioavailability of poorly water soluble drugs. European Journal of Pharmaceutics and Biopharmaceutics. 2008;70(2):439-444.

16. Passerini N, Albertini B, Perissutti B, Rodriguez L. Evaluation of melt granulation and ultrasonic spray congealing as techniques to enhance the dissolution of praziquantel. International journal of pharmaceutics. 2006;318(1):92-102.

17. Vilhelmsen T, Eliasen H, Schæfer T. Effect of a melt agglomeration process on agglomerates containing solid dispersions. International journal of pharmaceutics. 2005;303(1):132-142.

18. Thies C, Dos Santos IR, Richard J, Vandevelde V, Rolland H, Benoit JP. A supercritical fluidbased coating technology 1: Process considerations. Journal of Microencapsulation. 2003;20(1):87-96.

19. Tuleu C, Newton M, Rose J, Euler D, Saklatvala R, Clarke A, Booth S. Comparative bioavailability study in dogs of a self - emulsifying formulation of progesterone presented in a pellet and liquid form compared with an aqueous suspension of progesterone. Journal of pharmaceutical sciences. 2004;93(6):1495-1502.

20. Newton M, Petersson J, Podczeck F, Clarke A, Booth S. The influence of formulation variables on the properties of pellets containing a self - emulsifying mixture. Journal of pharmaceutical sciences. 2001;90(8):987-995.

21. Newton JM, Pinto MR, Podczeck F. The preparation of pellets containing a surfactant or a mixture of mono-and di-gylcerides by extrusion/spheronization. European journal of pharmaceutical sciences : official journal of the European Federation for Pharmaceutical Sciences. 2007;30(3):333-342.

22. Abdalla A, Mäder K. Preparation and characterization of a self-emulsifying pellet formulation. European Journal of Pharmaceutics and Biopharmaceutics. 2007;66(2):220-226.

23. Jannin V, Musakhanian J, Marchaud D. Approaches for the development of solid and semisolid lipid-based formulations. Advanced drug delivery reviews. 2008;60(6):734-746.

24. Sudheer P, Kumar N, Puttachari S, Shankar U. Approaches to development of solid-self micron emulsifying drug delivery system: formulation techniques and dosage forms-a review. Asian Journal of Pharmacy and Life Science. 2012;2231:4423.

25. Porter CJH, Charman WN. In vitro assessment of oral lipid based formulations. Advanced drug delivery reviews. 2001;50:S127-S147.

26. Pouton CW, Porter CJH. Formulation of lipid-based delivery systems for oral administration: Materials, methods and strategies. Advanced drug delivery reviews. 2008;60(6):625-637.

27. An J, Teoh JEM, Suntornnond R, Chua CK. Design and 3D Printing of Scaffolds and Tissues. Engineering. 2015;1(2):261-268.

28. Liu Z, Zhang M, Bhandari B, Wang Y. 3D printing: Printing precision and application in food sector. Trends in Food Science \& Technology. 2017;69(Part A):83-94.

29. Goyanes A, Martinez PR, Buanz A, Basit AW, Gaisford S. Effect of geometry on drug release from 3D printed tablets. International journal of pharmaceutics. 2015;494(2):657-663.

30. Khaled SA, Burley JC, Alexander MR, Yang J, Roberts CJ. 3D printing of five-in-one dose combination polypill with defined immediate and sustained release profiles. Journal of Controlled Release. 2015;217:308-314.

31. Basit AW, Gaisford S. 3D Printing of Pharmaceuticals: Springer International Publishing; 2018.

32. Norman J, Madurawe RD, Moore CMV, Khan MA, Khairuzzaman A. A new chapter in pharmaceutical manufacturing: 3D-printed drug products. Advanced drug delivery reviews. 2017;108:39-50.

33. Awad A, Trenfield SJ, Gaisford S, Basit AW. 3D printed medicines: A new branch of digital healthcare. International Journal of Pharmaceutics. 2018;548(1):586-596.

34. Awad A, Trenfield SJ, Goyanes A, Gaisford S, Basit AW. Reshaping drug development using 3D printing. Drug Discovery Today. 2018;23(8):1547-1555. 
35. Goyanes A, Scarpa M, Kamlow M, Gaisford S, Basit AW, Orlu M. Patient acceptability of 3D printed medicines. Int J Pharm. 2017;530(1-2):71-78.

36. Trenfield SJ, Goyanes A, Telford R, Wilsdon D, Rowland M, Gaisford S, Basit AW. 3D printed drug products: Non-destructive dose verification using a rapid point-and-shoot approach. Int J Pharm. 2018;549(1-2):283-292.

37. Moulton SE, Wallace GG. 3-dimensional (3D) fabricated polymer based drug delivery systems. Journal of controlled release : official journal of the Controlled Release Society. 2014;193:2734.

38. Alomari M, Mohamed FH, Basit AW, Gaisford S. Personalised dosing: Printing a dose of one's own medicine. International journal of pharmaceutics. 2015;494(2):568-577.

39. Fina F, Goyanes A, Gaisford S, Basit AW. Selective laser sintering (SLS) 3D printing of medicines. International Journal of Pharmaceutics. 2017;529(1):285-293.

40. Ghosh U, Ning S, Wang Y, Kong YL. Addressing Unmet Clinical Needs with 3D Printing Technologies. Advanced healthcare materials. 2018:e1800417.

41. Trenfield SJ, Awad A, Goyanes A, Gaisford S, Basit AW. 3D Printing Pharmaceuticals: Drug Development to Frontline Care. Trends in pharmacological sciences. 2018;39(5):440-451.

42. Fina F, Madla CM, Goyanes A, Zhang J, Gaisford S, Basit AW. Fabricating 3D printed orally disintegrating printlets using selective laser sintering. Int J Pharm. 2018;541(1-2):101-107.

43. Prasad LK, Smyth H. 3D Printing technologies for drug delivery: a review. Drug development and industrial pharmacy. 2016;42(7):1019-1031.

44. Alhnan MA, Okwuosa TC, Sadia M, Wan K-W, Ahmed W, Arafat B. Emergence of 3D printed dosage forms: opportunities and challenges. Pharmaceutical research. 2016;33(8):1817-1832.

45. Goyanes A, Buanz AB, Hatton GB, Gaisford S, Basit AW. 3D printing of modified-release aminosalicylate (4-ASA and 5-ASA) tablets. European Journal of Pharmaceutics and Biopharmaceutics. 2015;89:157-162.

46. Pietrzak K, Isreb A, Alhnan MA. A flexible-dose dispenser for immediate and extended release 3D printed tablets. European Journal of Pharmaceutics and Biopharmaceutics. 2015;96:380387.

47. Martinez PR, Goyanes A, Basit AW, Gaisford S. Fabrication of drug-loaded hydrogels with stereolithographic 3D printing. Int J Pharm. 2017;532(1):313-317.

48. Goyanes A, Buanz ABM, Basit AW, Gaisford S. Fused-filament 3D printing (3DP) for fabrication of tablets. International journal of pharmaceutics. 2014;476(1):88-92.

49. Khaled SA, Burley JC, Alexander MR, Yang J, Roberts CJ. 3D printing of tablets containing multiple drugs with defined release profiles. International Journal of Pharmaceutics. 2015;494(2):643-650.

50. Beck R, Chaves P, Goyanez A, Vukosavljevic B, Buanz A, Windbergs M, Basit A, Gaisford S. 3D printed tablets loaded with polymeric nanocapsules: an innovative approach to produce customized drug delivery systems. International Journal of Pharmaceutics. 2017.

51. Water JJ, Bohr A, Boetker J, Aho J, Sandler N, Nielsen HM, Rantanen J. Three - Dimensional Printing of Drug - Eluting Implants: Preparation of an Antimicrobial Polylactide Feedstock Material. Journal of pharmaceutical sciences. 2015;104(3):1099-1107.

52. Goyanes A, Det-Amornrat U, Wang J, Basit AW, Gaisford S. 3D scanning and 3D printing as innovative technologies for fabricating personalized topical drug delivery systems. Journal of controlled release. 2016;234:41-48.

53. Sun Y, Soh S. Printing tablets with fully customizable release profiles for personalized medicine. Advanced Materials. 2015;27(47):7847-7853.

54. Khan J, Rades T, Boyd BJ. Lipid-Based Formulations Can Enable the Model Poorly WaterSoluble Weakly Basic Drug Cinnarizine To Precipitate in an Amorphous-Salt Form During In Vitro Digestion. Molecular Pharmaceutics. 2016;13(11):3783-3793.

55. Kaukonen AM, Boyd BJ, Porter CJH, Charman WN. Drug solubilization behavior during in vitro digestion of simple triglyceride lipid solution formulations. Pharmaceutical research. 2004;21(2):245-253.

56. Mohsin K. Design of Lipid-Based Formulations for Oral Administration of Poorly WaterSoluble Drug Fenofibrate: Effects of Digestion. AAPS PharmSciTech. 2012;13(2):637-646. 
57. Thomas N, Holm R, Garmer M, Karlsson J, Müllertz A, Rades T. Supersaturated SelfNanoemulsifying Drug Delivery Systems (Super-SNEDDS) Enhance the Bioavailability of the Poorly Water-Soluble Drug Simvastatin in Dogs. The AAPS journal. 2013;15(1):219-227.

58. Khan J, Hawley A, Rades T, Boyd BJ. In Situ Lipolysis and Synchrotron Small-Angle X-ray Scattering for the Direct Determination of the Precipitation and Solid-State Form of a Poorly Water-Soluble Drug During Digestion of a Lipid-Based Formulation. Journal of pharmaceutical sciences. 2016;105(9):2631-2639.

59. Stillhart C, Imanidis G, Kuentz M. Insights into Drug Precipitation Kinetics during In Vitro Digestion of a Lipid-Based Drug Delivery System Using In-Line Raman Spectroscopy and Mathematical Modeling. Pharmaceutical research. 2013;30(12):3114-3130.

60. Williams HD, Sassene P, Kleberg K, Bakala - N'Goma JC, Calderone M, Jannin V, Igonin A, Partheil A, Marchaud D, Jule E. Toward the establishment of standardized in vitro tests for lipid - based formulations, part 1: method parameterization and comparison of in vitro digestion profiles across a range of representative formulations. Journal of pharmaceutical sciences. 2012;101(9):3360-3380.

61. Thomas N, Richter K, Pedersen TB, Holm R, Müllertz A, Rades T. In vitro lipolysis data does not adequately predict the in vivo performance of lipid-based drug delivery systems containing fenofibrate. The AAPS journal. 2014;16(3):539-549.

62. Griffin BT, Kuentz M, Vertzoni M, Kostewicz ES, Fei Y, Faisal W, Stillhart C, O’Driscoll CM, Reppas C, Dressman JB. Comparison of in vitro tests at various levels of complexity for the prediction of in vivo performance of lipid-based formulations: case studies with fenofibrate. European Journal of Pharmaceutics and Biopharmaceutics. 2014;86(3):427-437.

63. Munoz A, Guichard J, Reginault P. Micronised fenofibrate. Atherosclerosis. 1994;110:S45S48.

64. Vogt M, Kunath K, Dressman JB. Dissolution enhancement of fenofibrate by micronization, cogrinding and spray-drying: Comparison with commercial preparations. European Journal of Pharmaceutics and Biopharmaceutics. 2008;68(2):283-288.

65. Kleberg K, Jacobsen F, Fatouros DG, Müllertz A. Biorelevant media simulating fed state intestinal fluids: colloid phase characterization and impact on solubilization capacity. Journal of pharmaceutical sciences. 2010;99(8):3522-3532.

66. Kossena GA, Charman WN, Boyd BJ, Porter CJH. Influence of the intermediate digestion phases of common formulation lipids on the absorption of a poorly water-soluble drug. Journal of pharmaceutical sciences. 2005;94(3):481-492.

67. Warren DB, Anby MU, Hawley A, Boyd BJ. Real time evolution of liquid crystalline nanostructure during the digestion of formulation lipids using synchrotron small-angle X-ray scattering. Langmuir. 2011;27(15):9528-9534.

68. Larsen AT, Sassene P, Müllertz A. In vitro lipolysis models as a tool for the characterization of oral lipid and surfactant based drug delivery systems. International journal of pharmaceutics. 2011;417(1):245-255.

69. Kaukonen AM, Boyd BJ, Charman WN, Porter CJ. Drug solubilization behavior during in vitro digestion of suspension formulations of poorly water-soluble drugs in triglyceride lipids. Pharmaceutical research. 2004;21(2):254-260.

70. Phan S, Salentinig S, Prestidge CA, Boyd BJ. Self-assembled structures formed during lipid digestion: characterization and implications for oral lipid-based drug delivery systems. Drug Delivery and Translational Research. 2014;4(3):275-294. 